\title{
Infantile Colic
}

National Cancer Institute

\section{Source}

National Cancer Institute. Infantile Colic. NCI Thesaurus. Code C113481.

Paroxysms of irritability, fussing or crying that starts and stops without obvious cause in an infant up to four months of age without failure to thrive. Episodes last three or more hours per day for at least three days per week for at least on week. 